\title{
Author Index Volume 12 (2004)
}

The issue number is given in front of the page numbers.

Aldini, A., M. Bravetti and R. Gorrieri, A process-algebraic approach for the analysis of probabilistic noninterference

(2) $191-245$

Atluri, V., S.A. Chun and P. Mazzoleni, Chinese wall security for decentralized workflow management systems

(6) $799-840$

Backes, M., B. Pfitzmann, M. Waidner and M. Steiner, Polynomial liveness

Blyth, A., see Iheagwara, C.

Bravetti, M., see Aldini, A.

Broadfoot, P.J. and A.W. Roscoe, Embedding agents within the intruder to detect parallel attacks

Buchholz, F.P. and C. Shields, Providing process origin information to aid in computer forensic investigations

Buttyán, L., J.-P. Hubaux and S. Čapkun, A formal model of rational exchange and its application to the analysis of Syverson's protocol

$(3,4) 589-617$

(5) $777-798$

(2) $191-245$

$(3,4) 379-408$

(5) $753-776$

$(3,4) 551-587$

Čapkun, S., see Buttyán, L.

Cervesato, I., see Meadows, C.

Chander, A., D. Dean and J.C. Mitchell, Reconstructing trust management

Cho, T., S.-H. Lee and W. Kim, A group key recovery mechanism based on logical key hierarchy

Chun, S.A., see Atluri, V.

Dean, D., see Chander, A.

Devanbu, P., M. Gertz, A. Kwong, C. Martel, G. Nuckolls and S.G. Stubblebine, Flexible authentication of XML documents

Ding, Y., see Zhang, J.

Di Pierro, A., C. Hankin and H. Wiklicky, Approximate non-interference

Di Pietro, R., L.V. Mancini and A. Mei, Key management for high bandwidth secure multicast

Duggan, D., Type-based cryptographic operations

Durgin, N., P. Lincoln, J. Mitchell and A. Scedrov, Multiset rewriting and the complexity of bounded security protocols

$(3,4) 551-587$

(6) $893-931$

(1) $131-164$

(5) $711-736$

(6) $799-840$

(1) $131-164$

(6) $841-864$

(5) $737-751$

(1) $37-81$

(5) 693-709

$(3,4) 485-550$

(2) $247-311$

Gertz, M., see Devanbu, P.

(6) $841-864$

Gong, J., see Zhang, J.

(5) $737-751$ 
Gordon, A.D. and A. Jeffrey, Types and effects for asymmetric cryptographic protocols

Gorrieri, R., see Aldini, A.

Guttman, J., Guest editor's preface

Guttman, J.D., Authentication tests and disjoint encryption: A design method for security protocols

Guttman, J.D., F.J. Thayer and L.D. Zuck, The faithfulness of abstract protocol analysis: Message authentication

$(3,4) 435-483$

(2) $191-245$

(1) $1-1$

$(3,4) 409-433$

(6) $865-891$

Hankin, C., see Di Pierro, A.

Hopkins, B., see Ourston, D.

Hubaux, J.-P., see Buttyán, L.

Hughes, D. and V. Shmatikov, Information hiding, anonymity and privacy: a modular approach

(1) $37-81$

(2) $165-190$

$(3,4) 551-587$

(1) $3-36$

Iheagwara, C., A. Blyth and M. Singhal, Cost effective management frameworks for intrusion detection systems

(5) $777-798$

(5) $655-692$

Jajodia, S., see Wang, L.

Jeffrey, A., see Gordon, A.D.

Jha, S. and T. Reps, Model checking SPKI/SDSI

$(3,4) 435-483$

$(3,4) 317-353$

Kim, W., see Cho, T.

Kwong, A., see Devanbu, P.

(5) $711-736$

(6) $841-864$

Lee, S.-H., see Cho, T.

Lincoln, P., see Durgin, N.

Lowe, G., Analysing protocols subject to guessing attacks

Lowe, G., Defining information flow quantity

(5) $711-736$

(2) $247-311$

(1) $83-97$

$(3,4) 619-653$

Mancini, L.V., see Di Pietro, R.

(5) 693-709

(6) $841-864$

(2) $165-190$

Matzner, S., see Ourston, D.

Mazzoleni, P., see Atluri, V.

Meadows, C., P. Syverson and I. Cervesato, Formal specification and

(6) 799-840 analysis of the Group Domain Of Interpretation Protocol using NPATRL and the NRL Protocol Analyzer

Mei, A., see Di Pietro, R.

Micciancio, D. and B. Warinschi, Completeness theorems for the AbadiRogaway language of encrypted expressions

Mitchell, J., see Durgin, N.

Mitchell, J.C., see Chander, A.

(6) $893-931$

(5) $693-709$

(1) $99-129$

(2) $247-311$

(1) $131-164$

Nuckolls, G., see Devanbu, P.

(6) $841-864$

Ourston, D., S. Matzner, W. Stump and B. Hopkins, Coordinated Internet attacks: responding to attack complexity

(2) $165-190$ 
Pfitzmann, B., see Backes, M.

$(3,4) 589-617$

Reps, T., see Jha, S.

Roscoe, A.W., see Broadfoot, P.J.

$(3,4)$ 317-353

(3,4) 379-408

Scedrov, A., see Durgin, N.

(2) $247-311$

Schneider, S., Guest editor's preface

Shields, C., see Buchholz, F.P.

Shmatikov, V., Probabilistic analysis of an anonymity system

(3,4) 313-315

(5) $753-776$

$(3,4) 355-377$

(1) $3-36$

(5) $777-798$

Singhal, M., see Iheagwara, C.

Steiner, M., see Backes, M.

Stubblebine, S.G., see Devanbu, P.

$(3,4) 589-617$

(6) $841-864$

Stump, W., see Ourston, D.

Syverson, P., see Meadows, C.

(2) $165-190$

Thayer, F.J., see Guttman, J.D.

(6) $893-931$

Waidner, M., see Backes, M.

(6) $865-891$

Wang, L., D. Wijesekera and S. Jajodia, Cardinality-based inference control in data cubes

Warinschi, B., see Micciancio, D.

Wijesekera, D., see Wang, L.

Wiklicky, H., see Di Pierro, A.

$(3,4) 589-617$

(5) 655-692

(1) $99-129$

(5) $655-692$

(1) $37-81$

Zhang, J., J. Gong and Y. Ding, Research on automated rollbackability of intrusion response

(5) $737-751$

Zuck, L.D., see Guttman, J.D.

(6) $865-891$ 